\title{
ON THE SPACE-OF-LOOPS ISOMORPHISM
}

\author{
JAMES STASHEFF ${ }^{1}$
}

For $X$ an arc-wise connected topological space with base point $x_{0}$, let $\Omega X$ be the space of loops on $X$; that is, $\Omega X$ is the subset of the function space $X^{I}$ consisting of all paths $\lambda: I \rightarrow X$ such that $\lambda(0)$ $=\lambda(1)=x_{0}$, where $I$ is the unit interval. Let $|\Omega| X$ be the geometric realization of the singular complex of $\Omega X ;|\Omega| X$ is a CW-complex [5].

For arc-wise connected topological spaces $X$ and $Y$ with base points $x_{0}$ and $y_{0}$ respectively, denote by $\pi(X, Y)$ the set of homotopy classes of maps with base point of $X$ into $Y . \pi(X, Y)$ is in fact an invariant of the homotopy types of $X$ and $Y$, so we will use the most convenient representatives of the homotopy types in discussing $\pi(X, Y)$.

Recall that $\pi(X, Y)$ is known to have a natural group structure $[3$, p. 371] if it belongs to one of the following types:

(1) $X$ is a suspension space, or

(2) $Y$ is an $H$-space, or

(3) the dimension of $X$ is $\leqq 2 n-2$ where $Y$ is $(n-1)$-connected and either $X$ is a $\mathrm{CW}$-complex or $X$ is compact and $Y$ is a $\mathrm{CW}$ complex.

Our first theorem adds an approximate dual to (3), just as (1) and (2) are dual:

Theorem 1. If $X$ is an $(n-1)$-connected $C W$-complex and $\pi_{i}(Y)=0$ for $i>2 n-2$, then $\pi(X, Y)$ forms an abelian group, natural with respect to maps $X \rightarrow X^{\prime}$ and $Y \rightarrow Y^{\prime}$ where $X^{\prime}$ and $Y^{\prime}$ satisfy the same conditions.

With reference to [3] and the above list, we will say the pair $X, Y$ or the group $\pi(X, Y)$ is of type (4) under these conditions.

Peterson [6] has investigated the "dual" group of type (3) by means of the generalized suspension isomorphism. Continuing the duality, we show

Theorem 2. If $X$ is an $(n-1)$-connected $C W$-complex and $\pi_{i}(Y)=0$ for $i>2 n-2$, then $\pi(X, Y)$ is naturally isomorphic to $\pi(|\Omega| X, \Omega Y)$.

Theorem 2 can be interpreted as giving sufficient conditions for homotopy classes of maps of $|\Omega| X$ into $\Omega Y$ to coincide with loop homotopy classes in the sense of Kan [4, p. 41].

Presented to the Society April, 26, 1958; received by the editors January 23, 1959.

${ }^{1}$ During the preparation of this paper, the author was a Danforth Fellow and held an NSF Pre-doctoral Fellowship. 
Theorem 1 is proved in $\S 2$, the group structure is investigated in relation to the other types and a natural homomorphism $\Omega_{\sharp}: \pi(X, Y)$ $\rightarrow \pi(\Omega X, \Omega Y)$ is defined. $\S 3$ will prove the "Space-of-loops isomorphism."

I would like to thank J. F. Adams for many helpful discussions concerning $H$-spaces.

2. Before proving Theorem 1, we establish:

LeMma 1. If $Z$ is a $C W$-complex such that $\pi_{i}(Z)=0$ for $i<n$ and $i>2 n-2$ then $Z$ is an $H$-space; in fact, there exists a space $Z^{\prime}$ such that $Z$ has the homotopy type of $|\Omega| Z^{\prime}$.

Lemma 2. For such a $Z$, the $H$-space structure is unique up to homotopy.

Evidently both of these are well-known, though I am unable to assign an author to either. Lemma 2 is proved in [9] and Lemma 1 can be deduced from arguments there. We give a brief proof of the latter to lend insight into our basic approach.

Let $Z$ be represented by a finite Postnikov system $\left\{Z_{i}\right\}$, $i=n, \cdots, 2 n-2$. That is, $\pi_{j}\left(Z_{i}\right)=\pi_{j}(Z)$ for $j \leqq i$, and each $Z_{i}$ is the geometric realization of the singular complex of a fibre space over $Z_{i-1}$ with fibre $K\left(\pi_{i}(Z), i\right)$. The system and $Z$ are determined by $\pi_{i}(Z)$ and $k$-invariants $k^{i+1} \in H^{i+1}\left(Z_{i-1} ; \pi_{i}(Z)\right)$. We prove Lemma 1 by induction on $j$ where $\pi_{i}(Z)=0, i>j$.

Since $Z_{n}=K\left(\pi_{n}(Z), n\right)$, Lemma 1 is true for $j=n$, for $K\left(\pi_{n}(Z), n\right)$ has the same homotopy type as $|\Omega| K\left(\pi_{n}(Z), n+1\right)$.

Induction, $j-1 \Rightarrow j$ for $j \leqq 2 n-2$ : By induction, $Z_{j-1} \simeq|\Omega| Z_{j-1}^{\prime}$ for some $Z_{j-1}^{\prime}$. Thus $k^{j+1} \in H^{j+1}\left(|\Omega| Z_{j-1}^{\prime} ; \pi_{j}(Z)\right)$. Now $Z_{j-1}^{\prime}$ is $n$-connected, so we have the suspension isomorphism in cohomology:

$$
\sigma: H^{i+2}\left(Z_{j-1}^{\prime} ; \pi_{j}(Z)\right) \approx H^{i+1}\left(|\Omega| Z_{j-1}^{\prime} ; \pi_{j}(Z)\right)
$$

for $0<j+1<2 n[10$, p. $94 ; 8]$. Thus for $j \leqq 2 n-2, k^{i+1}=\sigma^{\prime} k^{i+2}$ for some ${ }^{\prime} k^{j+2} \in H^{i+2}\left(Z_{j-1}^{\prime} ; \pi_{j}(Z)\right)$, and so $Z_{j} \simeq|\Omega| Z_{j}^{\prime}$ where $Z_{j}^{\prime}$ is the fibre space determined by $Z_{j-1}^{\prime}$ and ' $k^{i+2}$.

Proof of Theorem 1 . Using the Postnikov system, we can represent $Y$ as a fibre space with fibre $Z$ and base $W$ where $\pi_{i}(Z)=\pi_{i}(Y)$, $i \geqq n$ and $=0, i<n$, and $\pi_{i}(W)=\pi_{i}(Y), i<n$ and $=0, i \geqq n$. Let $|Z|$ be the geometric realization of the singular complex of $Z$. By Lemma $1,|Z|$ is an $H$-space so that $\pi(X,|Z|)$ is a group, natural for maps $X^{\prime} \rightarrow X$ and $H$-maps $|Z| \rightarrow Z^{\prime}$.

Consider the map $\phi: \pi(X,|Z|) \rightarrow \pi(X, Y)$ induced by the inclusion $i: Z \rightarrow Y$ of $Z$ as the fibre through $y_{0}$. 
$\phi$ is onto: Since $X$ is $(n-1)$-connected, we can assume $X^{n-1}=x_{0}$. If $f: X \rightarrow Y$ then $f\left(X^{n-1}\right) \subset i(Z)$. Since $\pi_{n}(Y, Z) \approx \pi_{n}(W)=0$ we can deform $X^{n}$ into $i(Z)$ and similarly for $i>n$.

$\phi$ is 1-1: That is if $f: X \rightarrow Z$ is homotopic in $Y$ to $g: X \rightarrow Z$ then $f$ is homotopic to $g$ in $Z$. Since all the homotopies are relative to $x_{0}, f\left|X^{n-1} \simeq g\right| X^{n-1}$ in $Z$. Corresponding to each $k$-cell of $X$, there is a $k+1$-cell of $X \times I$. Again $\pi_{k+1}(Y, Z) \approx \pi_{k+1}(W)=0$ for $k \geqq n-1$ so we can deform the homotopy, skeleton by skeleton, into $i(Z)$. (We have actually shown $\phi$ is $1-1$ for $X(n-2)$-connected.) Although $Z$ may look like a space of loops on different spaces, the multiplications coincide by Lemma 2 so the group $\pi(X, Z)$ is uniquely determined. Since $Y$ does not determine $Z$ uniquely, we need to show further that the induced group structure on $\pi(X, Y)$ is independent of the choice of $Z$. This will be done while studying the naturality.

Naturality: That is, maps $f: Y \rightarrow Y^{\prime}$ where $\pi_{i}\left(Y^{\prime}\right)=0$ for $i>2 n-2$ or $g: X^{\prime} \rightarrow X$ where $X^{\prime}$ is an $(n-1)$-connected CW-complex induce homomorphisms $f_{*}: \pi(X, Y) \rightarrow \pi\left(X, Y^{\prime}\right)$ and $g^{*}: \pi(X, Y) \rightarrow \pi\left(X^{\prime}, Y\right)$. Consider first $g^{*}$. The diagram

$$
\begin{array}{cc}
\pi(X, Y) \stackrel{g^{*}}{\rightarrow} & \pi\left(X^{\prime}, Y\right) \\
\uparrow \phi & \uparrow \phi \\
\pi(X, Z) \underset{g^{*}}{\rightarrow} \pi\left(X^{\prime}, Z\right)
\end{array}
$$

is commutative, since $\phi$ is induced by $i$ and $(i \circ h) \circ g=i \circ(h \circ g)$ for $h: X \rightarrow Z . \phi$ is a homomorphism by definition and $g^{*}: \pi(X, Z)$ $\rightarrow \pi\left(X^{\prime}, Z\right)$ is known to be a homomorphism, hence $g^{*}: \pi(X, Y)$ $\rightarrow \pi\left(X^{\prime}, Y\right)$ is a homomorphism.

Before considering $f_{*}$, we give an alternative description of the group structure on $\pi(X, Y)$. J. H. C. Whitehead has proved [1, p. 288] that an $(n-1)$-connected CW-complex of dimension $\leqq 2 n-1$ is of the same homotopy type as a suspension space. Thus $\pi\left(X^{2 n-1}, Y\right)$ is a group of type (1). Consider $\eta: \pi(X, Y) \rightarrow \pi\left(X^{2 n-1}, Y\right)$ induced by the inclusion $X^{2 n-1} \subset X$. Just as for $\phi$, we prove

$\eta$ is onto: $f: X^{2 n-1} \rightarrow Y$ can be extended to all of $X$ since $\pi_{i}(Y)=0$ for $i>2 n-2$.

$\eta$ is 1-1: The obstructions to extending a homotopy vanish for the same reason. (In fact, $\eta$ is $1-1$, if $\pi_{i}(Y)=0$ for $i>2 n-1$.) Thus $\pi(X, Y)$ has a group structure induced by $\eta$ from that on $\pi\left(X^{2 n-1}, Y\right)$.

Since $X^{2 n-1}$ is not uniquely determined by the homotopy type of $X$ and further may be the suspension of two different spaces, the group $\pi(X, Y)$ has not yet been shown to be uniquely determined. This will 
follow from our next lemma which compares, for any given choices of $X^{2 n-1}$ as a suspension of a particular space and of $Z$, the corresponding structures on $\pi(X, Y)$ :

Lemma 3. $\eta$ and $\phi$ induce naturally isomorphic group structures on $\pi(X, Y)$.

Consider the diagram

$$
\begin{gathered}
\pi(X, Y) \stackrel{\eta}{\longrightarrow} \pi\left(X^{2 n-1}, Y\right) \\
\uparrow \phi \quad \phi^{\prime} \\
\pi(X,|Z|) \underset{\eta^{\prime}}{\longrightarrow} \pi\left(X^{2 n-1},|Z|\right)
\end{gathered}
$$

which is clearly commutative. $\eta^{\prime}, \phi^{\prime}$ are isomorphisms of both group structures, by naturality or by definition. Since the two structures agree in $\pi\left(X^{2 n-1},|Z|\right)[3]$, the induced ones agree in $\pi(X, Y)$. Since this is true for all choices of $X^{2 n-1}$ and $Z, \pi(X, Y)$ is well-defined and is an invariant of the homotopy types of $X$ and $Y$.

Revark. Since Hilton has shown $\pi(S, H)$ is an abelian group for $S$ a suspension space and $H$ an $H$-space, $\pi(X, Y)$ of type (4) is abelian. This agrees with the previous empirical evidence that two distinct natural group structures on $\pi(X, Y)$ will coincide and be abelian.

Returning to $f_{*}: \pi(X, Y) \rightarrow \pi\left(X, Y^{\prime}\right)$, consider the commutative diagram

$$
\begin{gathered}
\pi(X, Y) \stackrel{f_{*}}{\longrightarrow} \pi\left(X, Y^{\prime}\right) \\
\quad \downarrow \downarrow \eta \\
\pi\left(X^{2 n-1}, Y\right) \underset{f_{*}}{\longrightarrow} \pi\left(X^{2 n-1}, Y^{\prime}\right)
\end{gathered}
$$

corresponding to the situation for $g^{*}$ and $\phi . \eta$ is an isomorphism on both sides and $f_{*}: \pi\left(X^{2 n-1}, Y\right) \rightarrow \pi\left(X^{2 n-1}, Y^{\prime}\right)$ is known to be a homomorphism, hence $f_{*}: \pi(X, Y) \rightarrow \pi\left(X, Y^{\prime}\right)$ is. This concludes the proof of Theorem 1.

Since we know that two definitions of group structure for $\pi(X, Y)$ coincide, we might investigate the possibilities if $X, Y$ were also of type (1), (2) or (3). [Hilton has shown [3] that if $X, Y$ belongs to two of types (1), (2) or (3), then the group structures coincide.]

Lemma 4. If $X, Y$ is of type (4) and also (1), (2) or (3) the group structures coincide. 
Type (1). $X$ is a suspension space. We note that the definitions of type (1) and (2) coincide if they are both present, so that they coincide in $\pi(X,|Z|) . \phi$ is a homomorphism of the suspension structure by naturality and of type (4) structure by definition, hence the definitions coincide in $\pi(X, Y)$.

Type (2). $Y$ is an $H$-space. Dually to the above, we observe that the suspension and $H$-structures agree in $\pi\left(X^{2 n-1}, Y\right)$ and hence the type (4) and $H$-structures agree in $\pi(X, Y)$. Alternatively, it is possible to show that $|i|:|Z| \rightarrow Y$ is an $H$-map.

Type (3). We have $\operatorname{dim} X \leqq 2 m-2$ and $Y(m-1)$-connected. The situation is very simple for if $m \geqq n,|Y|$ is an $H$-space and we reduce to type (2), whereas if $m \leqq n X$ is a suspension space and we reduce to type (1).

In order to establish Theorem 2, we define $|\Omega|_{\#: \pi(X, Y)}$ $\rightarrow \pi(|\Omega| X, \Omega Y)$. For $f: X \rightarrow Y$, and $\lambda \in \Omega X$, let $\Omega *(f)(\lambda)=f \circ \lambda$ considering $\lambda$ as a map $I \rightarrow X$. There is a map $j:|\Omega| X \rightarrow \Omega X$ which induces isomorphisms of singular homology groups and homotopy groups. Define $|\Omega|_{\#(f)}$ as $\Omega_{\#}(f) \circ j$.

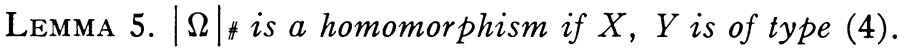

$|\Omega|_{\#}$ is a homomorphism if $\Omega_{\#}$ and $j^{*}$ are. The latter is a homomorphism by naturality of the $H$-structure, $\Omega Y$ being an $H$-space. That $\Omega_{\sharp}$ is a homomorphism can be seen from the following diagram:

$$
\begin{aligned}
& \pi(X, Y) \stackrel{\Omega_{\sharp}}{\longrightarrow} \pi(\Omega X, \Omega Y) \\
& \quad \phi \uparrow \\
& \pi(X, Z) \underset{\Omega_{\sharp}^{\prime}}{\longrightarrow} \pi(|\Omega| X, \Omega|Z|)
\end{aligned}
$$

$\Omega_{f^{\prime}}$ is a homomorphism since the loop multiplication on $\Omega|Z|$ is homotopic to that induced from the $H$-structure on $|Z| . \Omega \phi$ is clearly a homomorphism since it is induced by $\Omega_{\#}(|i|): \Omega|Z| \rightarrow \Omega Y$ which is an $H$-map. $\Omega \phi$ is in fact an isomorphism since $\pi_{i}(\Omega Y, \Omega|Z|)=0$ for $i \geqq n-1$ and $|\Omega| X$ is $(n-2)$-connected. Thus $\Omega_{\sharp}$ is a homomorphism.

3. In terms of the previous paragraph, to prove Theorem 2 , we need only show that $\Omega_{f^{\prime}}: \pi(X, Z) \rightarrow \pi(|\Omega| X, \Omega|Z|)$ is an isomorphism. We will prove the theorem by induction on $j$ where $\pi_{i}(Y)=0$ for $i>j \leqq 2 n-2$.

Special case. $n=1$ so $\pi_{i}(Y)=0$ for $i \geqq 0$ which means $\pi(X, Y)=0$ and $\pi(|\Omega| X, \Omega Y)=0$.

For $n>1$, we start with $j=n$ since $Z$ is $(n-1)$-connected. $|Z|$ is, 
up to homotopy type, $K\left(\pi_{n}(Y), n\right)$ so our theorem reduces to the known result

$$
H^{n}\left(X ; \pi_{n}(Y)\right) \approx H^{n-1}\left(\Omega X ; \pi_{n}(Y)\right) .
$$

Induction, $j-1 \Rightarrow j$ for $j \leqq 2 n-2$ : For any fibre space $E$ with fibre $F$ over base $B$ we have the following exact sequence [7, p. 199],

$$
\begin{aligned}
& \cdots \rightarrow \pi\left(X, \Omega^{n+1} B\right) \rightarrow \pi\left(X, \Omega^{n} F\right) \rightarrow \pi\left(X, \Omega^{n} E\right) \rightarrow \pi\left(X, \Omega^{n} B\right) \rightarrow \\
& \cdots \rightarrow \pi(X, \Omega B) \rightarrow \pi(X, F) \rightarrow \pi(X, E) \rightarrow \pi(X, B) .
\end{aligned}
$$

Applied to $Z_{j}$, of the Postnikov system of $|Z|$, we have

$$
\cdots \rightarrow \pi\left(X, \Omega Z_{j-1}\right) \rightarrow \pi(X, K) \rightarrow \pi\left(X, Z_{j}\right) \rightarrow \pi\left(X, Z_{j-1}\right)
$$

where $K=K\left(\pi_{j}(Z), j\right)$, but since $|Z| \simeq|\Omega| Z^{\prime}$ we can extend this and, writing $W=|\Omega| X, R=Z_{j}, S=Z_{j-1}$ and $K^{\prime}=K\left(\pi_{j}(Z), j+1\right)$, obtain

$$
\begin{aligned}
& \cdots \rightarrow \pi(X, \Omega S) \rightarrow \pi(X, K) \longrightarrow \pi(X, R) \longrightarrow \pi(X, S) \longrightarrow \pi\left(X, K^{\prime}\right) \rightarrow \cdots \\
& a] \downarrow \quad b] \downarrow \quad c] \downarrow \quad d] \downarrow \quad e] \downarrow \\
& \cdots \rightarrow \pi\left(W, \Omega^{2} S\right) \rightarrow \pi(W, \Omega K) \rightarrow \pi(W, \Omega R) \rightarrow \pi(W, \Omega S) \rightarrow \pi(W, K) \rightarrow \cdots
\end{aligned}
$$

where $K_{j}^{\prime}=K\left(\pi_{j}(Z), j+1\right)$.

All the sets involved are groups with horizontal arrows being homomorphisms. Lemma 5 applies to all the vertical arrows; they are

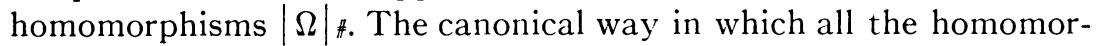
phisms are induced from maps makes all the squares commutative.

By induction we can assume that $a$ ] is an isomorphism for $j \leqq 2 n-1$ (since $\pi_{i}\left(\Omega Z_{j-1}\right)=0, i>j-2$ ) and that $\left.d\right]$ is an isomorphism for $j<2 n-1$. A special case of our theorem is the following

Suspension TheOREM. If $W$ is an $(n-1)$-connected space, the "suspension" map

$$
\sigma: H^{i}(W ; \pi) \rightarrow H^{i-1}(\Omega W ; \pi)
$$

is an isomorphism for $0<i<2 n-1$ and a monomorphism for $i=2 n-1$. $[10$, p. $94 ; 8]$.

Thus we also have that $b]$ is an isomorphism for $0<j<2 n-1$, monomorphism for $j=2 n-1$ and $e]$ is an isomorphism for $0<j+1$ $<2 n-1$, monomorphism for $j+1=2 n-1$. By the Five Lemma, $c$ ] is an isomorphism for $j \leqq 2 n-2$.

REMark. The final statement of the Suspension Theorem that $\sigma$ is monomorphic for $i=2 n-1$ can also be generalized.

Theorem 2a. If $X$ is an $(n-1)$-connected $C W$ complex, $\pi_{i}(Y)=0$ for $i>2 n-1$ and some choice of $|Z|$ is an $H$-space, then $|\Omega| \sharp: \pi(X, Y)$ $\rightarrow \pi(|\Omega| X, \Omega Y)$ is a monomorphism. 
For then we still have the exact sequences (7) (since $|Z|$ is an $H$ space, the map $Z_{j-1} \rightarrow K_{j}^{\prime}$ is an $H$-map and so $\pi\left(X ; Z_{j-1}\right) \rightarrow \pi\left(X, K_{j}^{\prime}\right)$ is a homomorphism) and the half of the Five Lemma [2, Lemma 4.4] necessary to prove $c$ ] monomorphic for $j=2 n-1$ requires only that $a$ ] be onto and that $b$ ] and $d$ ] be monomorphic. Modifying Theorem 2 to Theorem $2 \mathrm{a}$ throughout, the induction goes through.

The extra assumption that $|Z|$ is an $H$-space is necessary to make $\pi(X, Y)$ a group and the top line of (3) an exact sequence of homomorphisms.

If it is desired to have Theorem 2 in a more symmetric form, we need only observe that $\pi(|\Omega| X, \Omega Y)$ is isomorphic with $\pi(|\Omega| X,|\Omega| Y)$ since $j:|\Omega| Y \rightarrow \Omega Y$ induces isomorphisms of the homotopy groups and $|\Omega| X$ is a CW-complex. Whether $\pi(|\Omega| X, \Omega Y)$ is isomorphic with $\pi(\Omega X, \Omega Y)$ is related to the general problem of giving $\Omega X$ as a CW-complex, up to homotopy type. ${ }^{1}$

\section{Bibliography}

1. M. G. Barratt, Track groups II, Proc. London Math. Soc. (3) vol. 5 (1955) pp. 285-329.

2. S. Eilenberg and N. E. Steenrod, Foundations of algebraic topology, Princeton University Press, 1953.

3. P. J. Hilton, On divisors and multiples of continuous maps, Fund. Math. vol. 43 (1956) pp. 358-386.

4. D. M. Kan, On homotopy theory and c.s.s. groups, Ann. of Math. vol. 68 (1958) pp. $38-53$.

5. J. Milnor, The geometric realization of a semi-simplicial complex, Ann. of Math vol. 65 (1957) pp. 357-362.

6. F. P. Peterson, Generalized cohomotopy groups, Amer. J. Math. vol. 78 (1956) pp. 259-281.

7. - Functional cohomology operations, Trans. Amer. Math. Soc. vol. 86 (1957) pp. 197-211.

8. J.-P. Serre, Homologie singulière des espaces fibrés. Applications, Ann. of Math. vol. 54 (1951) pp. 425-505.

9. J. D. Stasheff, On homotopy associativity of $H$-spaces (to appear).

10. H. Suzuki, On the Eilenberg-MacLane invariants of loop spaces, J. Math. Soc. Japan vol. 8 (1956) pp. 93-101.

Princeton University and

Mathematical Institute, OXford

${ }^{1}$ Added in proof. This problem has recently been solved by J. Milnor. 